Anna Wilk*

\title{
ÜBER DIE POLITISIERUNG DES ANTIGONE-MYTHOS BEI ROLF HOCHHUTH ${ }^{1}$
}

\begin{abstract}
„Manchmal hilft es ja, hunderte von Kilometern weit wegzufahren oder hunderte von Jahren zurückzugehen, in eine Vergangenheit, die wir nur durch Sagen und Mythen kennen, um zu sehen, was man da findet [...]: Sich selbst. " (Wolf 1988, S. 11)
\end{abstract}

Christa Wolf

„Mythen sind geronnene kollektive Erfahrungen, zum andern ein Esperanto, eine internationale Sprache, die nicht mehr nur in Europa verstanden wird" (Müller 1992) meint Heiner Müller. Die Geschichte von Antigone und ihrer Schwesterliebe ist so alt wie die Welt und trotzdem stachelt sie die Interpreten zu immer neuen Deutungsbemühungen an. Der Antigone-Stoff erfuhr im Laufe der Jahrhunderte zahlreiche Bearbeitungen und Inszenierungen. Im 20. Jahrhundert wird er auch in $\mathrm{Zu}-$ sammenhang mit den beiden Weltkriegen gebracht, wie z. B. im expressionistischen Drama Antigone Walter Hasenclevers aus dem Jahre 1917, in der Erzählung Rolf Hochhuts Die Berliner Antigone (1963) oder im Drama Bertolt Brechts Die Antigone des Sophokles. Nach der Hörderlinschen Übertragung für die Bühne bearbeitet (1948). Rolf Hochhuth, der durch sein 1963 publiziertes Drama Der Stellvertreter bekannt geworden ist, in dem er das Thema des Holocausts und der Passivität der Kirche und des Papstes Pius XII. aufgreift, verlegt die Handlung des antiken Stoffes in die Zeiten des NS-Regimes. Die Berliner Antigone setzt sich mit dem Thema des Nationalsozialismus und der conditio humana auseinander. Anhand dieses Werkes lässt sich analysieren, welchen Transformationen ein Mythos unterliegen kann.

Den Verweis auf den Antigone-Mythos gibt es bereits im Titel, es ist aber keine Antigone des griechischen Tragikers, es ist eine Berliner Antigone. Dadurch

* Dr. Anna Wilk, Universität Łódź, Institut für Germanische Philologie, Pomorska 171/173, 90-236 Łódź. E-Mail: anna.wilk@uni.lodz.pl

${ }^{1}$ Dieser Artikel wurde bereits in der englischen Fassung veröffentlicht: Anna Zaorska, About the Politicization of the Antigone Myth by Rolf Hochhuth. In: Małgorzata Budzowska, Jadwiga Czerwińska (Hrsg.): Ancient Myths in the Making Culture, Frankfurt am Main 2015. 
wird es einerseits deutlich, dass der Text Hochhuths einerseits an die Tradition der Antike anknüpft, anderseits auch, dass der Stoff modernisiert worden ist. Was der Autor übernimmt, ist das Handlungschema von Sophokles, er vergegenwärtigt aber den Stoff und ändert den Handlungsort, die Figurennamen, die Sprache. Ein Novum ist auch die literarische Gattung: Hochhuth schreibt kein Drama, sondern eine Novelle mit einem allwissenden Erzähler. Die Handlung ist nicht chronologisch aufgebaut und besteht neben der äußeren Handlung aus der Innensicht, aus den Gedanken, Erinnerungen, Erlebnissen von zwei Hauptprotagonisten. Hochhuth lässt somit einen Perspektivenwechsel zu. Dieser Perspektivenwechsel bleibt allerdings aufgrund der Figurenkonstellation begrenzt, weil es zwischen den Protagonisten keine Zwiegespräche gibt, weil es zu keiner offenen Konfrontation kommt.

Die Titelfigur lernen wir kennen, als sie vor Gericht steht. Die Berliner Antigone heißt Anne, was, wie Detlef Brennecke bemerkt, eine Anspielung auf den Namen Antigone sein kann: Anne - An(tigo)ne (vgl. Brennecke 1987, S. 48). Anne ist eine junge Medizinstudentin, die trotz des Verbots ihren Bruder, den Staatverräter, bestattet. Ihr Bruder bleibt im Text - was nicht ohne Bedeutung ist - nur ein namenloser Offizier, der bei Stalingrad gekämpft hat. Er wird zum Tode verurteilt, denn

[d]er Mann, statt dankbar zu sein, daß er als Schwerverwundete mit einem der letzten Flugzeuge aus dem Kessel von Stalingrad ausgeflogen worden war, hatte nach seiner Genesung schamlos erklärt, nicht die Russen, sondern der Führer habe die 6. Armee zugrunde gerichtet (Hochhuth 2006, S. 6).

Die Leiche des Gehenkten - wie ihn oft im Laufe des Gerichtsverfahrens der Staatanwalt nennt - sollte der Anatomie übergegeben werden. Anne wollte dies verhindern, deswegen entführte sie den Körper ihres Bruders und hat ihn auf dem Invalidenfriedhof in Berlin beigesetzt. Sie will den ,»Verbleib « ihres toten Bruders' (Hochhuth 2006, S. 6) auch dann nicht preisgeben, als ihr die Todesstrafe droht. Sie nimmt das Risiko in Kauf, ,zermürbt und leise, beharrte [sie] auf ihrer Lüge“ (Hochhuth 2006, S. 6). Was Hochhuth noch von Sophokles übernommen hat, ist die ganze Figurenkonstellation: Anne ist mit Bodo, dem Sohn des Generalrichters verlobt. Der Generalrichter ist Vorsitzender im Prozess, er soll auch über eine adäquate Strafe entscheiden, dementsprechend ist er im Text Hochhuths eine Machtinstanz.

Im Gegensatz zur Antigone des Sophokles ist Anne keine Heroin. Ihr Handeln resultiert weder aus politischen noch aus religiösen Gründen. Eberhard Hermes bemerkt zurecht, dass sie oft inkonsequent handelt, sie ,zerbricht fast an ihrer inneren Schwäche und Todesangst' (Hermes 1992, S. 160). Man kann die Formulierung riskieren, dass sie sich vielmehr intuitiv verhält. Auch wenn der Priester versucht, ihr klar zu machen, dass, ein Unbestatteter nach christlicher Auffassung nicht ruhe- 
los bleibe' (Hochhuth 2006, S. 12), hält sie an ihrem Vorsatz fest. Sie ist allerdings nicht so stark wie ihr mythisches Vorbild. Noch vor der Beerdigung ihres Bruders ist Antigone von Sophokles sich dessen bewusst, mit welcher Strafe sie rechnen muss. Dennoch hat sie die Entscheidung getroffen, das Risiko auf sich zu nehmen. Wenn Kreon beabsichtigt, beide Schwestern zu bestrafen, weist sie Ismene $\mathrm{ab}$, um sie zu beschützen. Und wenn Ismene dennoch die Schuld ihrer Schwester teilen will, betont sie, dass sie mit dem Todesgedanken zurechtgekommen ist. Im Gegensatz zu Antigone hat Anne die Todesstrafe nicht erwartet und die Drohungen seitens des Richters haben sie innerlich gebrochen. Im Brief an ihren Verlobten zwingt sie sich, zu schreiben, dass sie es nicht für sinnlos hält, was sie getan hat und weswegen sie jetzt sterben muss (vgl. Hochhuth 2006, S. 11). Außerdem hat Anne Angst vor dem Tod, in einem Moment der Schwäche hat sie ein Gnadengesuch eingereicht. Es soll zum Nachdenken animieren, dass Anne das Gnadengesuch erst dann eingereicht hat, als alle ihr nahen Personen bereits tot waren (vgl. Hochhuth 2006, S. 9). Während der erste Teil der Handlung auf die Geschehnisse im Gerichtssaal konzentriert ist, fokussiert sich der zweite auf die Gefängnishaft Annes. Dabei wird explizit gezeigt, wie die Hauptheldin mit der drohenden Todesstrafe klarzukommen versucht, wie sie die ganze Situation analysiert. Die Gegenwart und die Rückblickperspektive werden dabei nach wie vor vermischt.

Anne zweifelt oft am Sinn ihrer Opferung. Wenn bei ihr die Daseinsbegierde überwiegt, wäre sie manchmal sogar bereit aufzugeben, wenn das nicht mit der Exhumierung des toten Bruders verbunden wäre. Es soll betont werden, dass ihr Handeln eher auf ihren Bruder und auf die familiären Verhältnisse konzentriert ist. Es ist kein Kampf um eine Ideologie oder ein Versuch des Widerstandes gegen das Regime Hitlers. Antigone von Sophokles erwähnt dagegen den Rang der Rechte Gottes; der Tod scheint ihr nicht schrecklicher zu sein als ein Leben in der Schande. Um Anne herum breitet sich der Tod aus, was ihr hilft an ihrer Entscheidung festzuhalten. Der Generalrichter sieht als einen mildernden Umstand „die seelische Zerrüttung: der Verurteilung des Bruders sei bekanntlich der Freitod ihrer Mutter gefolgt" (Hochhuth 2006, S. 8). Als die Hauptfigur erfährt, dass ihr Bräutigam Suizid begangen hat, empfindet sie ihr Leben nicht mehr lebenswert, aber auch dann spürt sie noch Angst. Mit der Verlängerung der Erwartung auf die Todesstrafe erlebt Anne psychische Qualen. Im Text wird betont, dass sie nicht ganz bereit ist, zu sterben:

Manchmal entrissen ihre Toten, der Freund, die Mutter, der Bruder, Anne ihrer Angst und bewirkten, daß das Unvorstellbare, ihr eigenes Totsein, vorstellbar wurde ohne Entsetzen, ja eben als die wahre verfälschte Freiheit. In solchen Momenten war sie bereit. In den Nächten, wenn sie lag, überwog ihre Daseinsbegierde (Hochhuth 2006, S. 16).

Anne agiert, im Gegensatz zu Antigone, auch nicht religiös. Wie es Detlef Brennecke formuliert: Im christlichen Sinn fromm ist sie nicht (vgl. Brennecke 1987, 
S. 50). Sie flüchtet sich in die Einsicht, daß allein der Tod uns beschützen kann. Der Tod, nicht Gott‘ (Hochhuth 2006, S. 16).

Anne wurde zum Tode verurteilt. Die zynische Anordnung Hitlers lautet: die Angeklagte solle ,in eigener Person der Anatomie die Leiche zurückerstatten (Hochhuth 2006, S. 7). Ihr Tod soll aber nicht nur als Strafe verstanden werden, ihre Verurteilung soll vielmehr eine Abschreckungsfunktion erfüllen: „Die Angeklagte sollte enthauptet und der Anatomie zur Abschreckung jener Medizinstudenten »überstellt werden«, die vermutlich bei der Beseitigung der Leiche ihres Bruders geholfen haben“ (Hochhuth 2006, S. 7). Im Text gibt es auch einen Hinweis auf die Studentenrevolte in München:

Hier in der Reichshauptstadt, unter den schadenfrohen Augen des Diplomatischen Korps, das hatte Hitler noch angefügt, sollte nicht geräuschvoll nach ungefährlichen Querulanten unter den Studenten gefahndet werden: peinlich genug, daß im Frühjahr die feindliche Presse von der Studentenrevolte in München Wind bekam, weil Freislers Volksgerichtshof zwar schlagartig, aber doch zu laut damit aufgeräumt hatte (Hochhuth 2006, S. 7).

Freislers Volksgerichtshof hat Sophie und Hans Scholl sowie einige der „Mittäter" in den Tod geschickt. In dieser Passage kommt es zum Ausdruck, wie die nationalsozialistische Gewaltherrschaft funktioniert hat. Während man noch versuchen könnte, Kreon zu rechtfertigen, der als Staatbeschützer erscheinen könnte - er versucht den Staatsverräter Polineykes zu bestrafen, der um Macht zu erobern seine Heimat, Theben angegriffen hat - so fehlt eine solche Art der Rechtfertigung bei Hitler ganz (Brennecke 1987, S. 50). Seine Herrschaft beruht nur auf Angst, Gewalt und Bedrohung, jede Art von Kritik ist nicht gestattet, jede Opposition soll niedergeschlagen werden. Auch dann, wenn die Kritik eines namenlosen, bei Stalingrad kämpfenden Soldaten, nicht gegen Staat, sondern gegen Hitler gerichtet ist.

Es scheint, dass im Text Hochhuths Kreon fehlt (vgl. Helmes 2011, S. 91), bzw. dass wir es mit zwei Kreon-Figuren zu tun haben. Der Generalrichter, der sich dem geltenden Gesetz des Dritten Reiches nicht widersetzt und Anne zum Tode verurteilt, könnte einer sein. Er folgt blind dem geltenden Gesetz und den Anordnungen Hitlers. Der Generalrichter wagt es nicht einmal zu gestehen, dass sein Sohn Bodo mit jenem ,Mädchen, d[er] Schwester eines Hochverräters' (Hochhuth 2006, S. 7), heimlich verlobt ist. Allerdings ist der Generalrichter kein Despot und Tyrann, wie Kreon im Text von Sophokles, denn er hat keine Macht, er übt nur Macht aus. Zu Beginn der Erzählung hofft er sogar, Anne retten zu können, was ihn fast zu einer positiven Gestalt machen könnte. Es kann aber nicht aus dem Auge geraten, dass seine Haltung trotzdem despotische Charakterzüge trägt. Während des Prozesses droht er der Angeklagten:

Sie können sich 24 Stunden überlegen, ob Ihre Helfershelfer in der Anatomie die Leiche Ihres Bruders dort wieder vorfinden - oder ob die Mitwisser durch Einlieferung Ihrer Körpers, Kopf vom Rumpf getrennt, darüber aufgeklärt werden sollen, daß wir Nationalsozialisten jeden defätistischen Ungehorsam rücksichtslos ausmerzen. (Hochhuth 2006, S. 9) 
Allerdings trifft der Generalrichter keine eigenständigen Entscheidungen, er gehorcht nur den Befehlen und Anordnungen, somit vertritt er nur den Diktator. Die andere Figur, die dem Kreon-Muster entspricht, wäre also Hitler selbst. Er ist zwar keine Hauptfigur im Text und wird oft nur im Hintergrund des Geschehens erwähnt, seine Rolle lässt sich jedoch nicht unterschätzen. Hitler ist diejenige Person, die das ganze Geschehen in Gang bringt, er ist Despot und Tyrann. Im Gerichtsaal findet man eine „Büste des Führers“, eine „kolossale Bronze“ (Hochhuth 2006, S. 6). Was man nicht übersehen kann: durch seine Befehle ist er auch im Laufe des Textes ständig präsent. Seine Präsenz, auch wenn sie zeitweise scheinbar fehlt, prägt den ganzen Text. Zum Schluss erweist sich aber, dass nur der Generalrichter alle Konsequenzen tragen wird. Bodo begeht an der Ostfront in einem russischen Bauernhaus Selbstmord, denn er glaubt, Anne sei bereits tot. Daraufhin zeichnet Hitler den Generalrichter mit der höchsten Stufe des Kriegsverdienstkreuzes aus. Kreon im Text von Sophokles hatte mindestens die Möglichkeit, seinen Fehler zu begreifen, musste aber daraufhin eine Strafe auf sich nehmen. Hitler kann durch die fehlende Konfrontation mit Anne seinen Entschluss nicht bereuen. Das betont nur seinen Despotismus, denn er ,bleibt unangefochten in seinem Führerhauptquartier und hat es nicht nötig, seine Entscheidung Anne gegenüber zu begründen“"(Hermes 1992, S. 168).

Der Generalrichter wird zur einer tragischen Figur, in der sich der innere Konflikt wiederspiegelt: einerseits will er die Verlobte seines Sohnes vor dem Tode bewahren, andererseits rät er aber Bodo, seine Verlobung nicht öffentlich zu verkündigen, und letztendlich entscheidet er über die Todesstrafe für Anne. Sein Konflikt besteht im Widerstreit der Interessen: familiäre vs. politische Rücksichten, Moral vs. Staatsräson. Eberhard Hermes bemerkt zurecht, dass eine Kreon-Haimon-Szene in Antigone von Sophokles eine andere Funktion hat, als die Szene am Bahnhof, kurz bevor Bodo an die Ostfront geschickt wird (vgl. Hermes 1992, S. 168). Diese Szene verdeutlicht vor allem einen Vater-Sohn-Konflikt und die innere Zerrissenheit des Generalrichters. Außerdem kommt es zum Treffen an einem anderen Zeitpunkt, als Antigone alias Anne noch nicht verurteilt wurde. Dass die Todesstrafe verhängt wurde, erfährt Bodo erst aus einem Abschiedsbrief Annes, als er bereits an der Ostfront ist. In dieser Szene wird dennoch sichtbar, wie es an Kommunikation zwischen den Figuren fehlt. Denn im ganzen Text gibt es keine Kommunikation. Die Protagonisten beharren stillschweigend auf ihrem Standpunkt, ohne den Partner/Gegner zu überzeugen zu versuchen. Anne wagt nicht einmal vor Gericht ihre Handlungsweise zu rechtfertigen oder zu erklären. Wie Hermes weiter bemerkt, gibt es ebensowenig eine Konfrontation zwischen Antigone und dem Machtinhaber:

Entsprechend der Handlungsstruktur ist die Personenkonstellation verändert. Das AntigoneDrama hat als Zweifigurenstück ein doppeltes Zentrum, weil sich die Handlung zwischen den beiden gegensätzlichen Hauptgestalten in der Form des Streits entwickelt. In der Novelle 
gibt es keinen Streit, sondern nur die Selbstherrlichkeit und Menschenverachtung auf der politischen Ebene, wo die Entscheidungen getroffen werden, und die Ohnmacht, die Angst, das Lavieren, den kleinen Ehrgeiz bei den Menschen, welche die Folgen jener Entscheidungen tragen müssen und dabei entweder die Rolle des Opfers oder des Mitläufers spielen. Deshalb ist die Figur der Anne - anders als Antigone - hier keine Widerstandsheldin, die Kreon selbstbewußt gegenübertritt, sondern eine in der Einsamkeit bleibende, in der Todesangst schwach werdende, für die Öffentlichkeit unbedeutende Frau. Kreon, ihr Gegenüber, aber ist in zwei unterschiedliche Figuren aufgespalten. Für Hitler ist Anne lediglich einer der „ungefährlichen Querulanten unter den Studenten“ (S.7, Z.21/2), deren Fall ohne Aufsehen zu erledigen ist (Hermes 1992, S. 169).

Was ins Auge fällt: Sowohl Anne als auch den Generalrichter kennzeichnet Passivität. Er selbst gibt zu, dass er ,vielleicht auch nicht mehr imstande gewesen [wäre], die Delinquentin [...] aus der angelaufenen Vernichtungsmaschinerie zurückreißen" (Hochhuth 2006, S. 15). Anne dagegen war handlungsfähig nur bis zum Gerichtsprozess.

Offen bleibt die Frage, wie Antigone alias Anne stirbt: ob sie wie ,eine Heilige“ in den Tod geht, oder ob sie ein Foto in den Händen hält, „um für ihre Augen einen Halt zu finden" (Hochhuth 2006, S. 18). Dass Anne zusammen mit sechs anderen Frauen stirbt, verleiht ihrem Tod eine kollektive Bedeutung. Anne ist eins der zahlreichen Opfer des NS-Regimes und sie ist nicht imstande, irgendwelchen Widerstand zu leisten. Ihr Tod zerstört nicht die Strukturen des NS-Staates, während der Tod Antigones Kreon und seine Welt erschüttert hat. Bei Hochhuth sehen Hitler und sein Stab zum Schluss einen Film mit der Hinrichtung der Männer, die am 20. Juli 1944 versuchten, das Regime zu stürzen. Der Text endet mit einem Epitaph:

Die Berliner Anatomie

erhielt in den Jahren 1939-1945

die Körper

von 269 hingerichteten Frauen“ (Hochhuth 2006, S. 18)

Anne stirbt so namenlos wie ihr Bruder und die anderen hingerichteten Frauen. Man könnte ergänzen: so namenlos wie andere Opfer des NS-Regimes. Interessanterweise agiert Anne nicht politisch, sie wird aber in die politische Vernichtungsmaschinerie des NS-Regimes hineingezogen.

Rolf Hochhuth basiert in seinem Werk auf Fakten. Seine Novelle ist aber keine historische Dokumentation, sondern bleibt literarische Fiktion. Der Text trägt die Widmung ,Für Marianne‘ (Hochhuth 2006, S. 5). Marianne Heinemann war die Ehefrau von Rolf Hochhuth (Kreuzer 2006, S. 68). Ihre Eltern, die in einer sozialistischen Familientradition standen, sind tragisch gestorben. Die Mutter, Rose Schlösinger, wurde 1943 in Berlin enthauptet, weil sie zur Widerstandsgruppe um Arvid und Mildred Harnack gehörte. Der Vater, Bodo Schlösinger, hat sich in einem russischen Bauernhaus erschossen, er wollte die ganze Schuld auf sich neh- 
men und seine Frau vor dem Tode retten. Diese Geschehnisse sind zum Bestandteil von Hochhuths Novelle Die Berliner Antigone geworden.

„Antigone wird [...] zum Symbol des Widerstands gegen autokratische Politik und der Rechte des Individuums gegenüber kollektivem Zwang. Im erstem Weltkrieg erhebt Walter Hasenclever 1917 in seiner Antigone, einem der ersten Dramen des Expressionismus, die Stimme gegen den Krieg [...]“ (Hermes 1992, S. 17). Im Text Hochhuths ist Anne keine politisch oder moralisch agierende Person, in diesem Sinne leistet sie keinen Widerstand, sie ist eher Opfer des Nationalsozialismus. Hermes pointiert zutreffend:

Wie Bertolt Brecht weiß natürlich auch Rolf Hochhuth, daß, "die große Figur des Widerstands im antiken Drama nicht die Kämpfer des Deutschen Widerstands, die uns am bedeutendsten erscheinen müssen, repräsentiert.

Worauf es diesem Autor vielmehr ankommt, ist „die Rolle der Gewaltanwendung“ [...] eine Gewaltanwendung, die sich vor allem in der Sprache der Gewalttäter manifestiert (Hermes 1992, S. 58).

Hochhuth schildert die Wirklichkeit des totalitären Systems und die Nichtachtung der Menschenwürde. Ironisch beschreibt er, wie die zum Tode verurteilten Personen „Pakete“ (,eine Fachbezeichnung für Patienten mit geringer Lebenserwartung") (Hochhuth 2006, S. 15) genannt werden. Mit dem Tod wird der Leser Allerdings schon von der ersten Seite an konfrontiert:

Die Bezeichnung Massengrab war verboten worden. Die Reichsregierung pflegte die Toten eines Gemeinschaftsgrabes mit besonders tröstlichem Aufwand beizusetzen: nicht nur waren Geistliche beider Konfessionen und ein namhafter Parteiredner, sondern auch noch ein Musikzug des Wachbataillons und eine Fahnenabordnung hinzugezogen worden (Hochhuth 2006, S. 5).

Im Text wird überdies betont, wie die Nationalsozialisten mit den politischen Gegnern verfahren sind: sie sollen nicht so laut aufgeräumt werden, denn alles soll den Anschein erwecken, dass es gemäß dem geltenden Recht geschieht. Die Gewaltanwendung kommt vor allem im Stillschweigen der Protagonisten zum Ausdruck, „Manchmal ist Schweigen der lauteste Schrei“. Rolf Hochhuth hat durch die Aktualisierung der antiken Vorlage bewiesen, dass die conditio humana sich im Laufe der Jahrhunderte nicht wesentlich verändert hat.

In den Mythen liegt großes Identifikationspotential. Die Mythen werden demnach nicht als märchenhafte, sagenhafte Erzählungen verstanden, vielmehr als Geschichten, die überzeitliche, moralische Wahrheiten vermitteln. Das ist wahrscheinlich ein Grund dafür, warum uns die Mythen bis ins 21. Jahrhundert hinein begleiten und warum sie Faszination sowohl seitens der Schriftsteller als auch der Literaturwissenschaftler hervorrufen. Denn auch das große Potenzial des Antigone-Mythos liegt in der Aktualität der Motive, die diese Geschichte zum Ausdruck bringt. Christa Wolf schreibt: 
In diesem Sinne, als Modell, das offen genug ist, um eigene Erfahrungen aus der Gegenwart aufzunehmen, das einen Abstand ermöglicht, den sonst oft nur die Zeit bringt, dessen Erzählungen fast märchenhaft, sehr reizvoll und doch so wirklichkeitsgesättigt sind, daß wir Heutige uns in den Verhaltensweisen seiner handelnden Personen erkennen können - in diesem Sinne scheint mir der Mythos brauchbar zu sein für den heutigen Erzähler, die heutige Erzählerin. Er kann uns helfen, uns in unserer Zeit neu zu sehen, er hebt Züge hervor, die wir nicht bemerken wollen und enthebt uns der Alltagstrivialität. Er erzwingt uns auf besondere Weise die Frage nach dem Humanum, um die es ja, glaube ich, bei allem Erzählen geht. (Wolf 1988, S. 14f.)

Es lässt sich dabei nicht übersehen, dass viele Schriftsteller die Mythen oft in den Wende- und Kriegszeiten sowie in den Zeiten der totalitären Systeme aufgegriffen haben, um einige Probleme in einer Parabel ausdrücken. Inge Stephan behauptet, dass die Rolle der Mythen vor allem dann an Bedeutung gewinnt, wenn nationale Identität bedroht ist. Leszek Kołakowski stellt zurecht fest, dass wir durch Mythen die Zeit anhalten wollen. Wir glauben, dass das, was bereits vergangen ist, in den Werten und Ideen aufrechterhalten bleibt (Kołakowski 1972, S. 18).

\section{LITERATURVERZEICHNIS}

Brennecke D. (1987), Rolf Hochhuths Novelle Die Berliner Antigone. In: Wolff R. (Hrsg.), Rolf Hochhuth. Werk und Wirkung, Bonn.

Helmes G. (2011), Antigone in Nazi-Deutschland. In: Bauer M., Jäger M. (Hrsg.), Mythopoetik in Film und Literatur, München.

Hermes E. (1992), Der Antigone-Stoff. Sophokles, Anouilh, Brecht, Hochhuth, Stuttgart.

Hochgeschurz M. (1988), Christa Wolfs Medea. Voraussetzungen zu einem Text. Mythos und Bild, Berlin. Hochhuth R. (2006), Die Berliner Antigone, Stuttgart.

Kołakowski L. (1972), Obecność mitu, Warszawa.

Kreuzer H. (2006), Die Berliner Antigone. Nachwort. In: Hochhuth R., Die Berliner Antigone, Stuttgart. Müller H. (1992), Krieg ohne Schlacht. Leben in zwei Diktaturen, Köln.

Wolf Ch. (1988), Von Kassandra zu Medea. Impulse und Motive für die Arbeit an zwei mythologischen Gestalten. In: Hochgeschurz M., Christa Wolfs Medea. Voraussetzungen zu einem Text. Mythos und Bild, Berlin.

Anna Wilk

\section{ABOUT THE POLITICIZATION OF THE ANTIGONE MYTH BY ROLF HOCHHUTH}

(Summary)

The Antigone myth is one of the most famous myths in the history of literature. History of sisterly love is as old as the human civilization, although it still inspires literary scholars to look for new interpretations. Rolf Hochhuth converts mythical theme into Second World War story. Die Berliner Antigone looks at the National Socialism and asks about conditio humana. Likewise Hochhuth's 
Antigone rejects human laws and buries her dead brother - nameless officer sentenced to death for his "shameless" remark: It was Hitler, not Russians, who destroyed the $6^{\text {th }}$ Army at the Stalingrad. Interestingly Anne, alias Antigone, is not motivated by politics or religion but nevertheless she's still dragged into political machinations and extermination system.

Heiner Müller wrote in his biography: 'myths are clotted collective experiences, or esperanto - an international language, that is understood not only in Europe'. Basing on Hochhuth's story one can analyze transformations of a myth and functions attributed to it only to notice that models human behavior are basically the same. In his novel German writer is only referencing ancient myth showing readers, through modernization, how timeless the theme is.

Keywords: myths in the literature, Antigone, Rolf Hochhuth, Die Berliner Antigone 